\title{
Navigation Doppler Lidar sensor for precision altitude and vector velocity measurements flight test results
}

\author{
Diego Pierrottet ${ }^{\mathrm{a}}$, Farzin Amzajerdian ${ }^{\mathrm{b}}$, Larry Petway ${ }^{\mathrm{b}}$, Bruce Barnes ${ }^{\mathrm{b}}$, George Lockard ${ }^{\mathrm{a}}$, and Glenn Hines ${ }^{\mathrm{b}}$ \\ ${ }^{\mathrm{a} C}$ Coherent Applications, Inc., Hampton VA, 23666

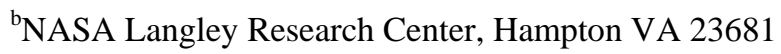

\begin{abstract}
An all fiber Navigation Doppler Lidar (NDL) system is under development at NASA Langley Research Center (LaRC) for precision descent and landing applications on planetary bodies. The sensor produces high-resolution line of sight range, altitude above ground, ground relative attitude, and high precision velocity vector measurements. Previous helicopter flight test results demonstrated the NDL measurement concepts, including measurement precision, accuracies, and operational range. This paper discusses the results obtained from a recent campaign to test the improved sensor hardware, and various signal processing algorithms applicable to real-time processing. The NDL was mounted in an instrumentation pod aboard an Erickson Air-Crane helicopter and flown over vegetation free terrain. The sensor was one of several sensors tested in this field test by NASA's Autonomous Landing and Hazard Avoidance Technology (ALHAT) project.
\end{abstract}

Key words: Lidar, Doppler, Navigation

\section{INTRODUCTION}

The Navigation Doppler Lidar (NDL) is under development at NASA Langley Research Center (LaRC) in support of the Autonomous Landing and Hazard Avoidance Technology (ALHAT) project. ALHAT is developing the NDL to support precision pin point landing on the lunar surface. The NDL is a multipurpose instrument capable of measuring high precision velocity vectors, precision altitude, and ground relative attitude including roll, pitch, angle of attack and side-slip angle. These vehicle state measurements can then be used by the guidance, navigation and control systems to navigate towards a predefined surface location with very high accuracies.

This report summarizes the results obtained from a field test of the second generation implementation of the sensor. Significant improvements have been made in hardware to reduce mass, volume, and power to the breadboard tested in the first ALHAT field test. ${ }^{\mathrm{i}}$. In addition, the hardware components have been upgraded to support automated signal processing that is necessary for real-time line of sight (LOS) range and velocity measurements, including the autonomous removal of measurement ambiguities. These improvements are a major developmental milestone as they bring the sensor closer to a technology readiness level 6 (TRL6). The NDL's first field test was primarily a proof of concept experiment to assess its capabilities. It was shown that the sensor is capable of very high accuracy measurements of LOS range and velocity. From LOS range measurements, altitude and attitude relative to ground (roll, pitch) were computed. From LOS velocity measurements, sideslip angle, and angle of approach were computed, velocity vector components were generated and the magnitudes of the vectors were compared to GPS measurements to excellent agreement.

This second field test was a very challenging campaign to equipment and personnel, due to the high summer temperatures in the California dessert, and the extreme vibration environment of the Erickson Air-Crane helicopter. The results presented here were obtained from flights that included navigation over various terrains, altitude and velocity profiles, and approach profiles to the target site. A description of the waveform, the system, and the first field test results were reported previously ${ }^{\mathrm{i}}$. Waveform description and measurement concepts are briefly repeated here for convenience. 


\section{WAVEFORM DESCRIPTION}

The lidar obtains high-resolution range and velocity information from a frequency modulated-continuous wave (FMCW) laser beam whose instantaneous frequency varies linearly with time. The amplitude of the waveform versus time is constant (cw). Figure 1 shows a description of the waveform's frequency content versus time. The blue sawtooth waveform represents the frequency content of the transmitted waveform, and the green trace simulates a received waveform. The shift in time of the received waveform is due to the time delay generated by the round trip time of flight of the laser beam to the target. The vertical shift represents the frequency change due to the Doppler effect caused by the moving vehicle relative to the target (ground). Immediately below these waveforms is the intermediate frequency (IF) between the transmitted and received waveforms as a function of time. In one full period of the waveform, two values of an IF are generated: one for the positive slope and one for the negative slopes of the waveforms. The IF generated during the positive slope is designated as the up-ramp frequency $\left(f_{i f}^{+}\right)$and the negative slope IF is the down-ramp frequency $\left(f_{i f}^{-}\right)$.

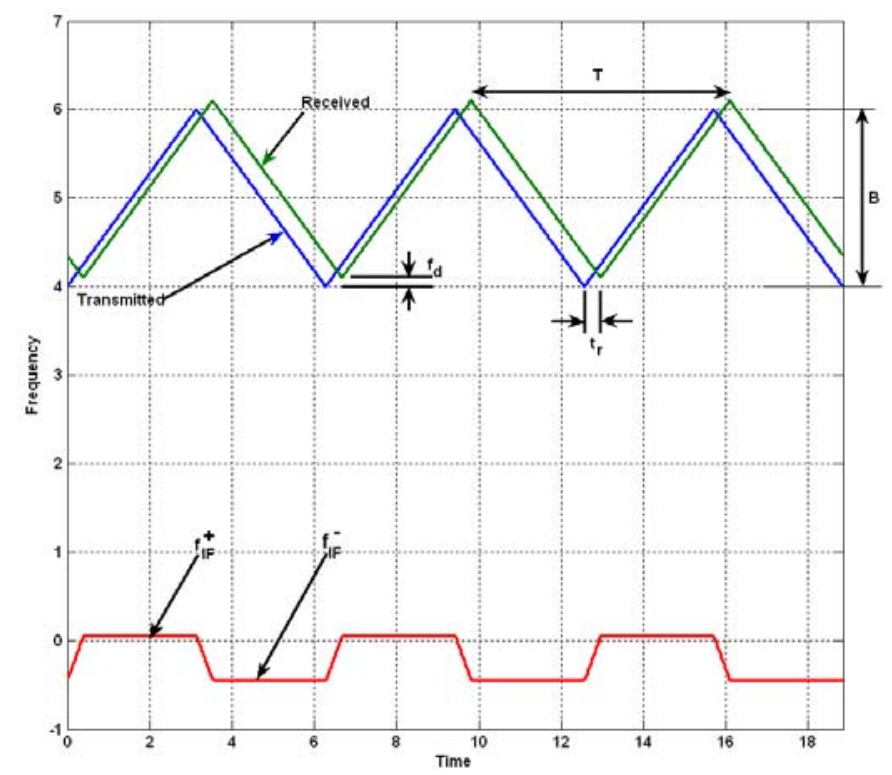

Figure 1 The green and blue curves represent the frequency content of the NDL waveform vs. time. Blue is the transmitted waveform, green is the received waveform and red is the difference, (intermediate frequency) between transmit and receive waveforms.

The NDL uses a homodyne configuration, in which a portion of the transmitted beam serves as the reference local oscillator (LO) for the optical receiver. The LO optical field mixes with the time delayed received field at the detector yielding a time varying IF as shown by the red trace in Fig. 1, and which is directly related to the target range by the equation

$$
f_{R}=\frac{4 R B}{T C}
$$

where $\mathrm{R}$ is the range to target, $\mathrm{B}$ is the modulation bandwidth, $\mathrm{T}$ is the waveform period and $\mathrm{c}$ is the speed of light. One can view equation 1 as a function of slope times a time delay $\Delta$, where the slope $m=2 B / T$, and the time delay for a particular range $\mathrm{R}$ is $\Delta t=2 R / \mathrm{C}$. Eq. (1) can also be written in terms of the up-ramp and down-ramp frequencies as 


$$
f_{R}=\frac{f_{i f}^{+}-f_{i f}^{-}}{2}
$$

Thus for known up- and down-ramp frequencies, and waveform slope, the LOS range is easily computed after equating the right side of Eq. 1 to the right side of Eq.2 and solving for R. For the case of a moving target, a Doppler frequency shift will be superimposed to this IF. The LOS Doppler frequency shift is simply

$$
f_{d}=\frac{f_{i f}^{+}+f_{i f}^{-}}{2}
$$

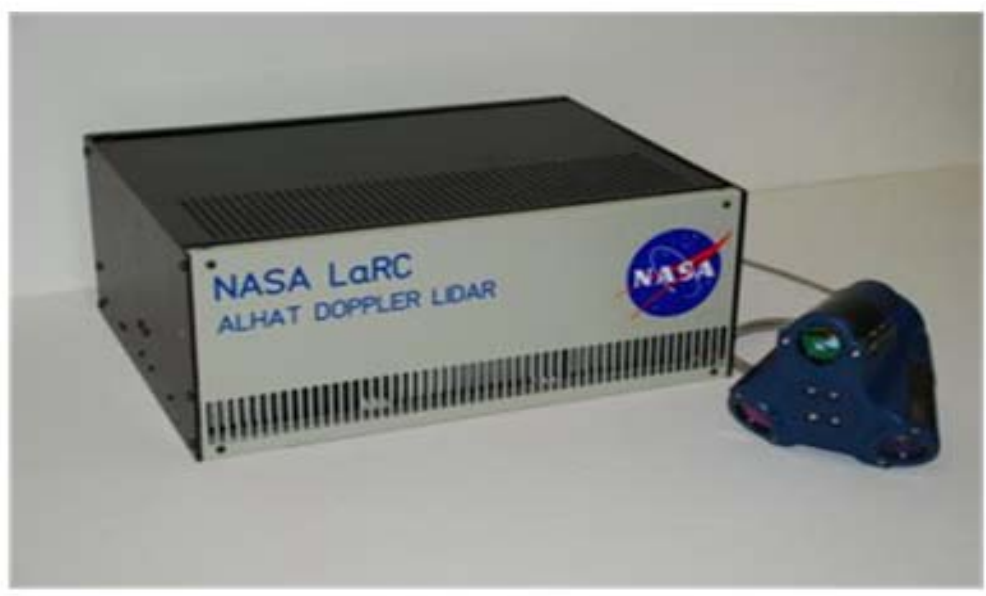

Figure 2 Doppler Lidar rack-mountable chassis and optical head. The support chassis is not shown.

The target radial velocity component is obtained from the equation

$$
v=\frac{f_{d} \lambda}{2 \cos \theta}
$$

Where $\lambda$ is the transmitter laser wavelength and $\theta$ is the angle between the target velocity vector and the lidar line of sight. To obtain horizontal and vertical velocity components, three measurements are made using three different sensor laser beams having a-priori pointing knowledge. The three independent yet instantaneous measurements provide three equations for the three unknown vector components vx, vy, and vz. This velocity vector has its origin at the NDL optical head and the $\mathrm{x}-, \mathrm{y}$ - and $\mathrm{z}$-axes are defined relative to the NDL optical head frame of reference.

\section{HARDWARE DEVELOPMENT}

For this implementation of the sensor, significant improvements have been made to the mass, volume, and power consumption. To reduce mass and volume, the oversized water-cooled heat sinks used in the first generation of the sensor were replaced with a single air-cooled heat sink and fans. The smaller heat sink was possible in part because the critical temperature dependent devices were independently thermally controlled using thermoelectric (TE) coolers and/or designing for higher temperature operations. Reduction in the cooling capacity needed was enabled by modifying the transmitter laser to operate at a low duty cycle. This also helped to significantly reduce the overall power consumption and total mass of the system.

The NDL is an all fiber system, meaning that the laser, amplifier, waveform modulator, and all beam train components are constructed from optical fibers or are integrated into a fiber-based configuration. Although most of the fiber components used to build this sensor are available as commercial-off-the-shelf (COTS) components, many components were custom built in-house in order to obtain the required performance and control of the sensor. One 
such component built for this generation of the sensor is the optical waveform synthesizer. This custom-built device provides precise knowledge of waveform parameters including modulation slope and timing information. As Figure 1 illustrates, each measured IF can be positive or negative, depending on the magnitude and direction of the Doppler shift. For example, a small positive Doppler shift will cause the up-ramp IF to be positive and the down-ramp IF to be negative, just as the IF (red) curve on Figure 1 shows. Likewise, a large positive Doppler shift will cause both up- and down-ramp IFs to be negative. Ambiguity in the measurement comes from not knowing if the IF is positive or negative. Although the magnitude will be correct, the wrong designation (phase) leads to large errors in range and velocity measurements as can be verified from equations (2) and (3). In addition to not having knowledge of the sign of the IF, one also does not have knowledge of the sign of the Doppler shift, i.e., one cannot tell if the target is moving towards or away from the observer. The waveform synthesizer that was developed in-house at LaRC provides precise control of the waveform parameters and allows programmable modulation schemes that greatly assist in removing Doppler ambiguities and frequency ambiguities from the measurements.

On the signal detection side, significant improvements were made to the optical receiver. Considerable effort was placed on improving the frequency response of the receiver, and reducing the noise floor. A new design was used in the amplification and filtering stages of the receiver that essentially flattened the frequency response over the bandwidth of interest. The improved frequency response enabled a lower gain than the previous receiver version did thus improving the signal dynamic range of the receiver. With lower gain and improved signal coupling, the noise floor was lowered, and increasing the signal to noise ratio (SNR) of the receiver without increasing the transmitter power.

A significant amount of effort was placed in the new data acquisition and signal-processing module. The new analog to digital conversion module has a 14-bit dynamic range, small footprint, and is connected directly to a FPGA that performs system control and signal processing. This acquisition/controller module was designed to fit within the NDL main chassis. Unfortunately, the module interface was not completed and tested in time for deployment into this field test, and so a replacement data-acquisition module was borrowed from the first generation system where the data is stored directly onto a hard drive for post processing. This data acquisition system is not capable of testing any of the real-time algorithms or control of the sensor and, therefore, results obtained from this field test had to be post processed. The ambiguity removal feature was hard-wired into the sensor processor and ran in an open loop configuration in order to evaluate the performance after the flight test. The results obtained from post flight data processing have proved very valuable in the development and performance optimization of the realtime algorithms.

\section{EXPERIMENTAL RESULTS}

The data analyzed and discussed in this report was obtained during Flight 4 . This flight was chosen because of the completeness of the data covering various terrains, altitudes, velocity profiles, and approach profiles to the target site. Flight 4 was an early morning takeoff when the air temperature was in the mid to high eighties. The sensor performed flawlessly for the duration of the flight providing a wealth of information on its operational performance. The following results were processed after the flight was completed since the real time signal acquisition and processing module was not deployed to this field test.

\section{Ambiguity Removal Algorithm}

The hardware developed and its supporting software algorithms were designed to function autonomously to provide the measured parameters in engineering units, and in real time. To test the functionality of the processing module the data of Flight 4 was batch processed and the results were evaluated. Altitude and velocity vector measurements require information from all three NDL LOS channels simultaneously. In the event that any one of the channels has measurement ambiguities then the error propagates to the final measurement value. There are still circumstances that can cause the ambiguity removal algorithm to fault, such as effects from false alarms. However, the most common faults occur when one of the IF (up- or down-ramp) for any one channel is experiencing a "zero crossing". "Zero crossing" refers to the time when a signal crosses from a positive value to a negative value in frequency. Near zero frequency (dc), signals are attenuated (low SNR) due to the ac-coupling of the receiver electronics, thus increasing the probability of false alarms. Backscatter clutter is also at its highest near dc, so identification of ambiguities is very difficult (positive vs. negative frequencies near zero). Details of the hardware and software developed to remove measurement ambiguities are documented internally at NASA and CAI, however the results presented here attest to the validity and success of development. Ambiguities were autonomously removed by the system, and applied to obtain high accuracy navigation states that are described next. 


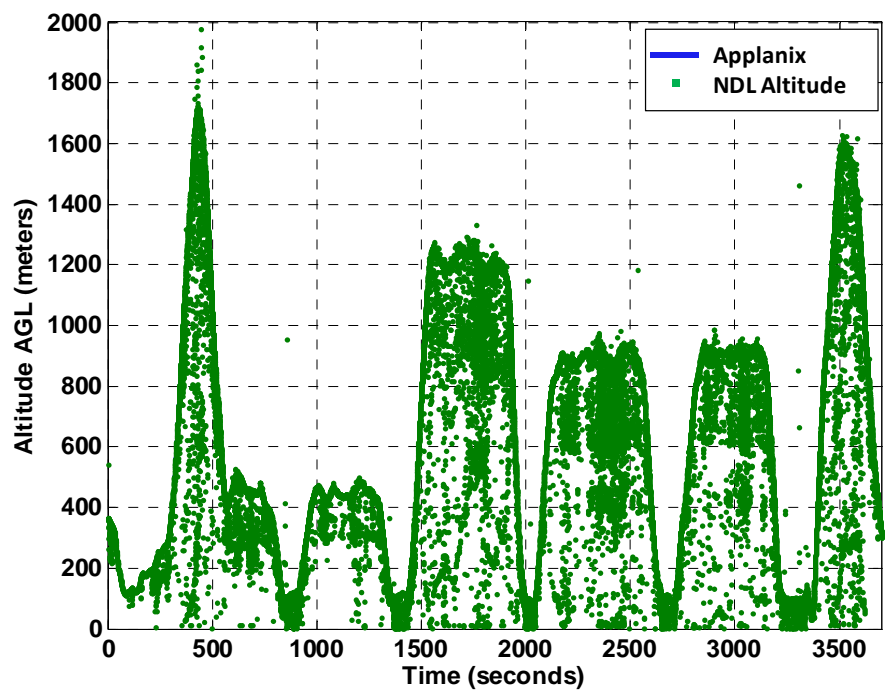

Figure 3 Altitude measurements obtained by the NDL. The agreement between the two instruments causes the Applanix results to hide behind the NDL data at this scale.

\section{Altitude}

Altitude measurements are obtained from the three independent LOS range measurements. The measured value is not the mean range, but a geometric computation of a line vector originating at the sensor, and parallel to the ground plane normal unit vector. The ground plane is defined by the three points on the ground created by the angular separation of the three LOS range measurements. This technique is more robust than a single range measurement of an altimeter pointing in the nadir direction since it reduces biases caused by rocks, hills, or craters. Figure 3 shows the unambiguous altitude measurements obtained using this method. The data is plotted together with the Applanix GPS altitude data. Because the Applanix measures altitude relative to sea level, a ground altitude bias is removed from the Applanix data before comparing to the above ground level (AGL) altitude measurements of the NDL. A

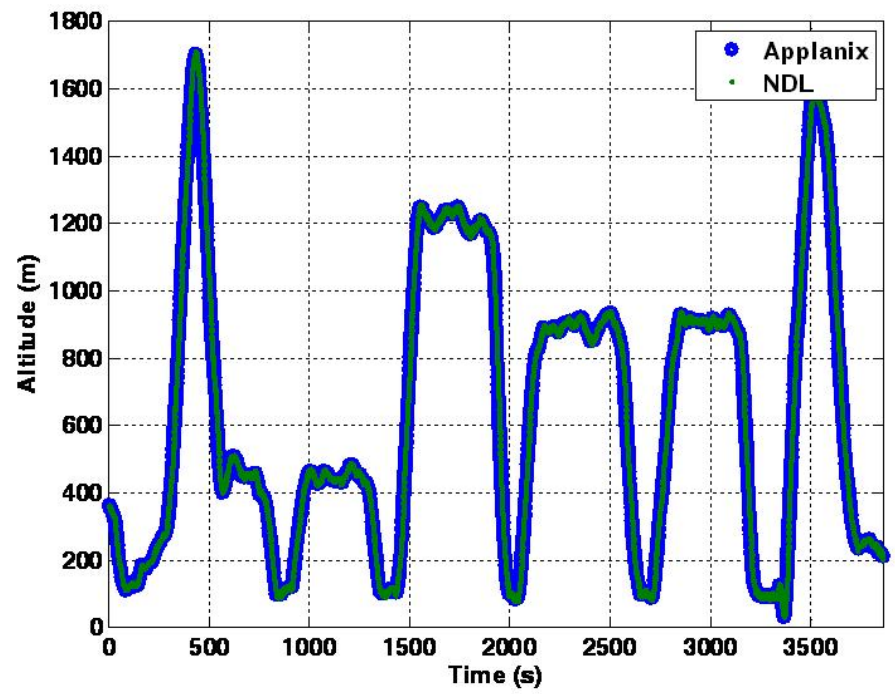

Figure 4 Filtered altitude measurements compared to GPS based Applanix 
bias of 673 meters was obtained near the start of the flight, during low altitude measurements over flat terrain and applied to the entire data set, without adjustments for ground elevation changes that are expected in various locations across the tested terrain. Because of the scale of the figure and the agreement between the two instruments, the Applanix data is not visible in the figure. The various outliers in the figure are caused by false alarms and zero crossings. Approximately $20 \%$ of the data was lost due to false alarms, primarily caused during zero crossings of the signals. The majority of the outliers can be removed using standard Kalman filters, or a similar recursive filter.

Figure 4 shows the filtered data plotted together with the Applanix data. The altitude profiles for the flight were chosen to simulate landing approach angles and velocities. The Erikson air crane helicopter set the limitations in altitude and speed, reaching a peak height of approximately 1697 meters, and a peak speed of approximately $58 \mathrm{~m} / \mathrm{s}$.

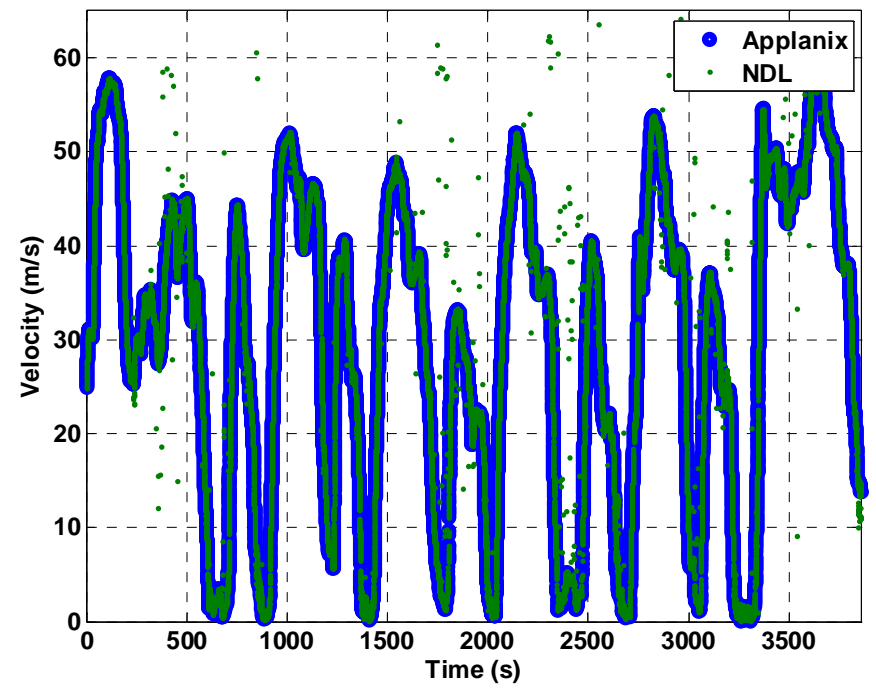

Figure 5 Velocity comparison shows excellent agreement between the two instruments.

\section{Velocity}

The NDL provides a very accurate measurement of vehicle velocity in relation to the ground. Measurements are made using the Doppler effect that is created by the moving body. A more thorough description of how the NDL measures vector velocity is provided in a previous paper (see Ref. $i$ ). Velocity magnitude comparison between the Applanix and the NDL provides a performance metric of the measurements. Figure 5 is a plot of the filtered data results comparing the two instruments. A data window of approximately 3700 seconds ( $>1$ hour) of Flight 4 containing over 74,000 NDL measurements was used to make the analysis and comparisons. The filter used to remove altitude outliers was used to identify outliers in the velocity data since both range and velocity are obtained from the same signals. Since each altitude measurement corresponds to the same velocity measurement in time, there is no need to use a velocity specific filter for this analysis. With most false alarms and zero crossing faults removed, a better comparison can be made to the Applanix results. The filter is used to remove outliers only, not to smooth the data in any way. This distinction is important because it allows a better quantitative performance comparison between the measurements from the Applanix and NDL.

Figure 6 is a is a 15 second window showing the velocity magnitude of both instruments (top) and its corresponding velocity discrepancies (bottom). Two noisy effects are noted in the measurements: high frequency velocity fluctuations and a low frequency discrepancy. Under controlled laboratory conditions, the accuracy and precision of the NDL was measured to less than one centimeter per second. Given this accuracy, the noisy appearance in the velocity measurements is not measurement error, but rather the measurements of actual velocities arising from the Erikson helicopter platform vibrations. 

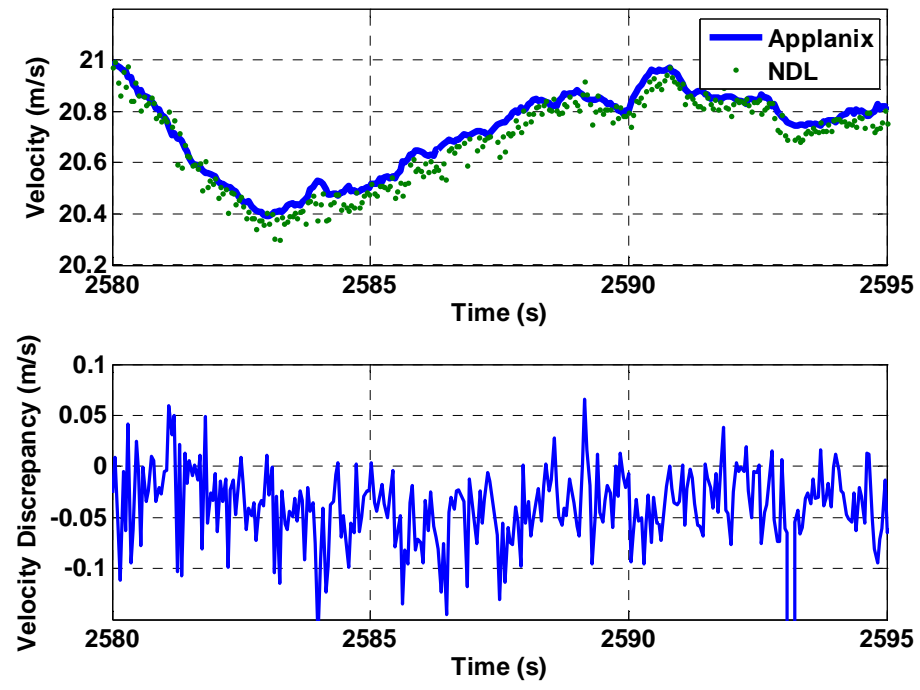

Figure 6 Data close-up of measured velocities (top) and velocity measurement discrepancy (bottom)

The slow varying discrepancies can be attributed to the physical separation of the two instruments relative to the center of mass of the helicopter. The recurrences of these discrepancies coincide with the helicopter maneuvers as witnessed by the high correlation in velocity discrepancy to attitude measurements, in particular to the pitch of the helicopter. Figure 7 is a plot of measurement discrepancy and the platform pitch measurements provided by the Applanix for all of Flight 4.

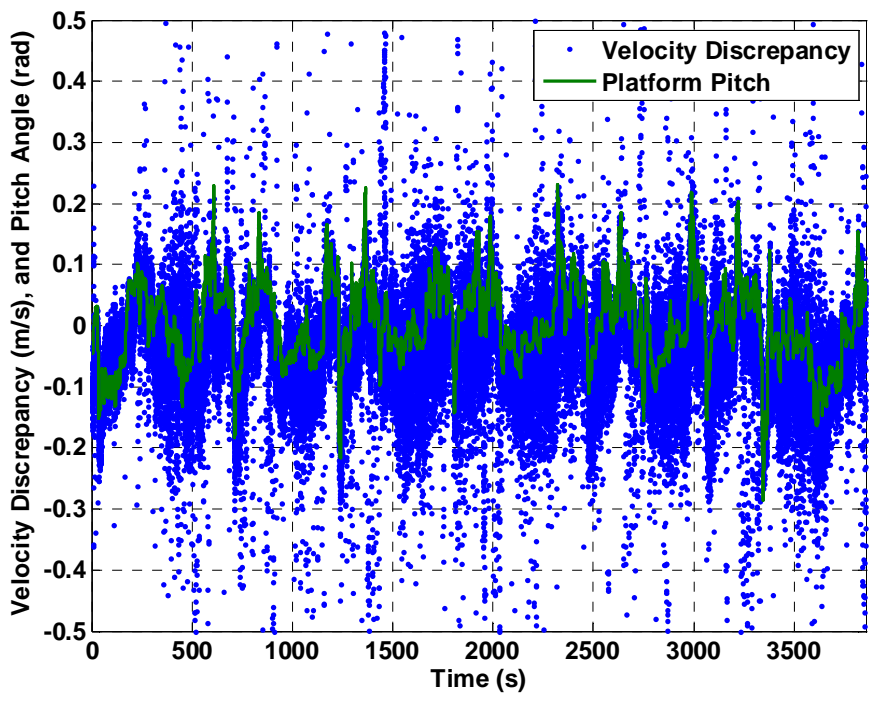

Figure 7 Superposition of velocity discrepancy with platform pitch show high correlation between the two. 


\section{Vector Velocity Components}

In addition to speed (velocity magnitude) measurements, the NDL provides high accuracy velocity vectors, computed at the sensor platform frame of reference. To compare the vector components of the NDL to those provided by the Applanix, vector transformations were made into a common frame of reference. Applying the transformation angles provided by the Applanix to the NDL velocity vectors, a one to one comparison of each component is made. Figures 8 are plots of the $\mathrm{x}$ (top left), $\mathrm{y}$ (top right), and $\mathrm{z}$-axis (bottom) velocity components of the two instruments.

As can be seen in these three plots, the velocity components obtained from the NDL are in excellent agreement with the velocity components of the Applanix. The mean velocity discrepancies are $0.7 \mathrm{~cm} / \mathrm{sec}, 0.4 \mathrm{~cm} / \mathrm{sec}$, and 2.7

$\mathrm{cm} / \mathrm{sec}$ for the $\mathrm{x}-, \mathrm{y}-$, and $\mathrm{z}$ - vector components respectively. The total mean velocity magnitude discrepancy is 3.5 $\mathrm{cm} / \mathrm{sec}$.
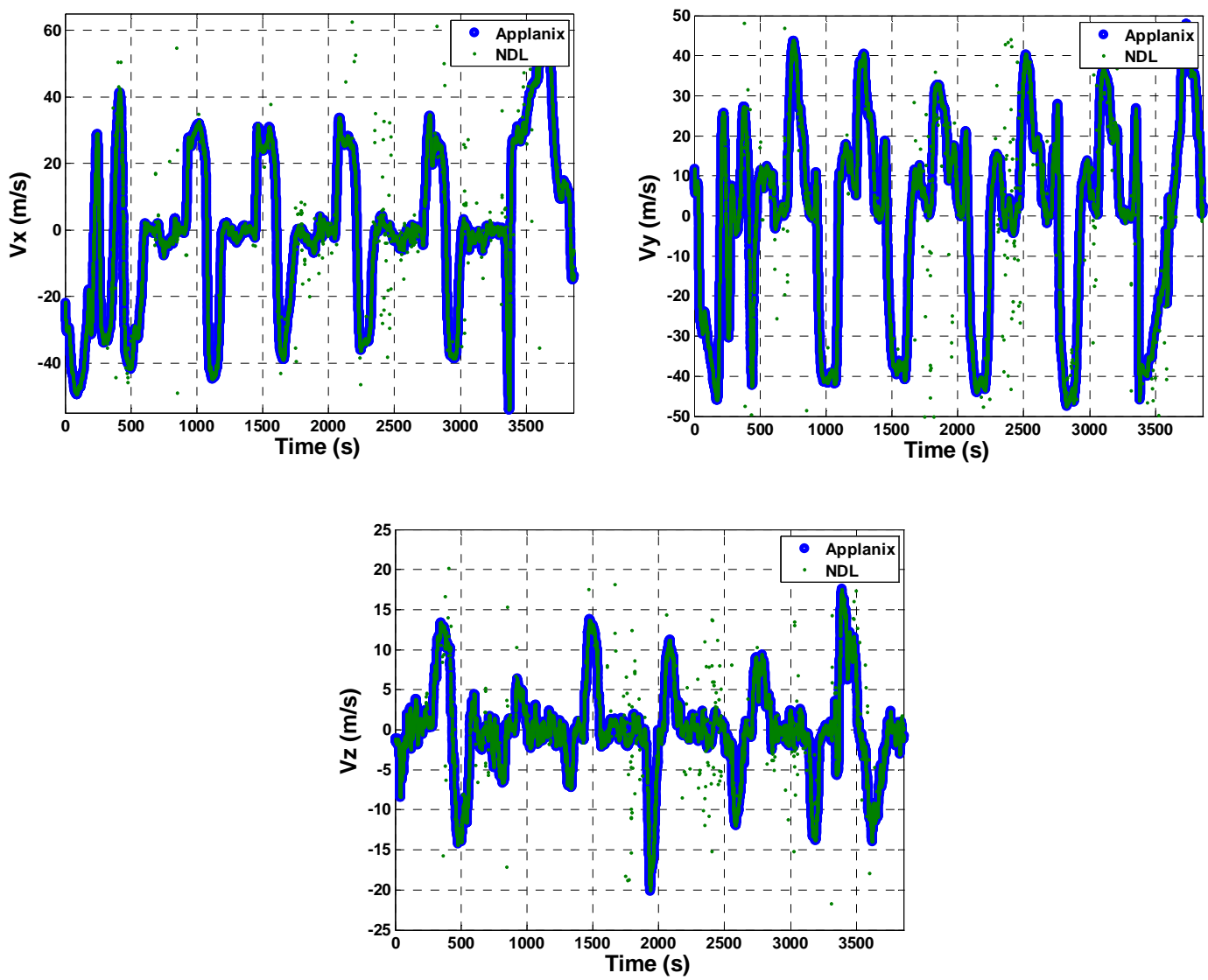

Figure 8 Velocity vector components compared to Applanix velocity components. 


\section{Platform Attitude}

Vehicle attitude measurements by the NDL are a product of the LOS range measurements, and are therefore relative to the ground plane generated by the three LOS range values. Figure 9 is a comparison of pitch angle between the NDL ground relative measurement and the pitch angle measured by the Applanix.

Figure 10 is a close-up look at the comparison. At the 2000 second mark, the altitude is approximately 88.5 meters during a descent; the speed is $35.6 \mathrm{~m} / \mathrm{sec}$ and decelerating. At this time the pitch angle discrepancy is the largest during this figure's 300 second window. The velocity discrepancies during this same window is highly correlated to the pitch discrepancies, suggesting that the discrepancy in pitch is not due to ground topography, but rather to sensor physical position on the platform. The agreement in pitch angle between the two measurements is very good, having a mean discrepancy of $10.7 \mathrm{mrad}$, and a standard deviation of $4 \mathrm{mrad}$ during this typical sample window of the flight.

A similar comparison is made for platform roll angle. Figure 11 is the close-up comparison of the two instruments for the same time window shown in Figure 10. The mean measurement discrepancy for this window in roll angle is $15.7 \mathrm{mrad}$, and the standard deviation is $6.8 \mathrm{mrad}$.

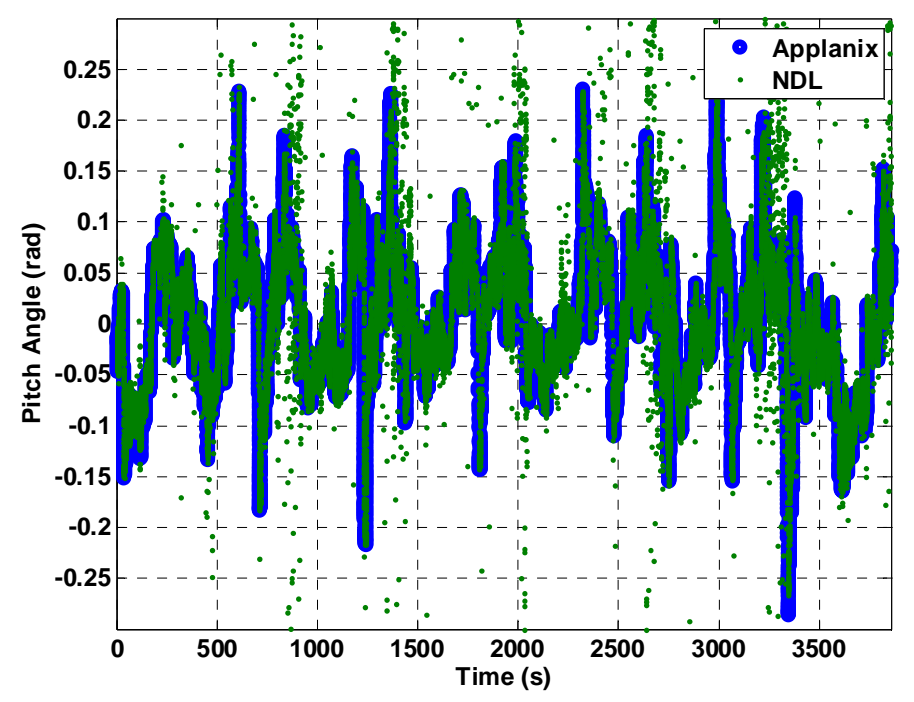

Figure 9 Pitch angle comparisons between the NDL ground relative measurements to the Applanix measurements. 

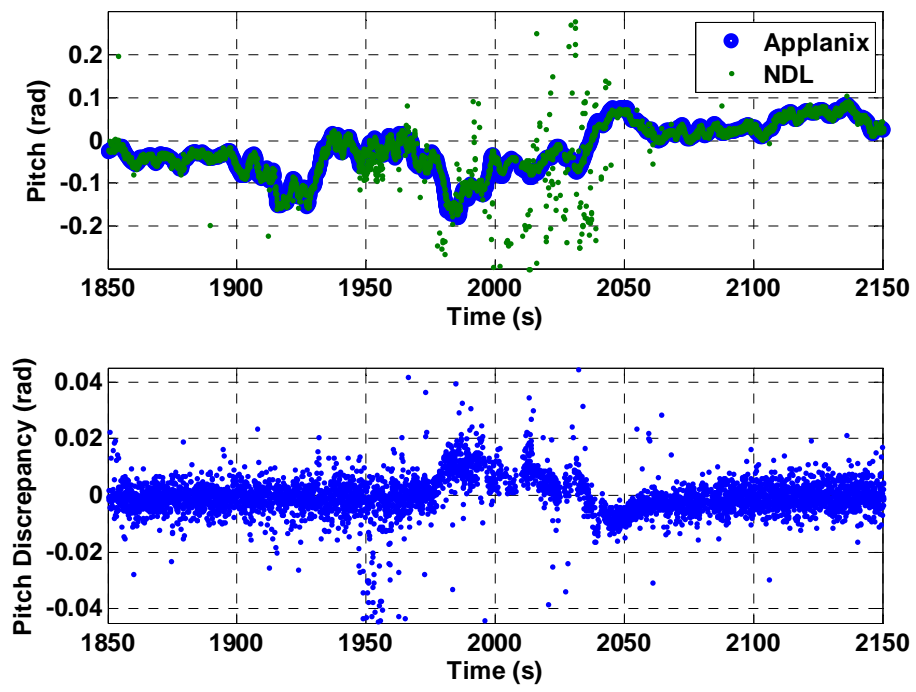

Figure 10 Close up of pitch angle (top) and measurement discrepancies (bottom).
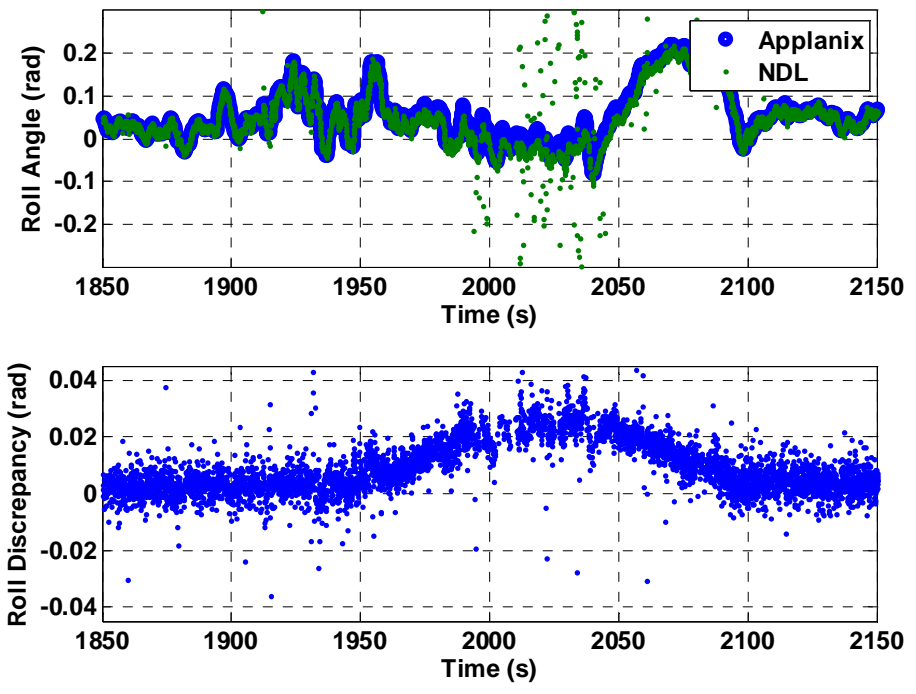

Figure 11 Close - up of roll angle (top) and angle measurement discrepancies (bottom). 


\section{Conclusions}

FT4 was a challenging experiment for the NDL in terms of overcoming the physical demands on the sensor, in addition to the complexity of the tests. As such, data was lost on some of the flights due to excessive shock and vibration that specifically affected the data storage devices, and extreme air temperatures that reached above 38 degrees $C$ and even higher inside the instrumentation pod. In spite of these problems, the NDL collected a wealth of data, which will be used beyond what was presented here. Although the external storage devices during one flight failed from the shock and vibration, the sensor hardware proved to be mechanically robust and reliable. Thermally, the sensor had a weakness in terms of operating under significant temperature differentials. For example, while sitting on the tarmac, ground air conditioning was provided to the instrumentation pod to keep the sensors, lasers, computers, etc., from overheating. Once the aircraft was ready for flight with the pod doors closed, the temperature inside the pod climbed steadily while taxiing and awaiting lift off from the tarmac. During flight with the instrument hatch open, the pod temperature would cool. Although the sensor was tested to operate up to $50^{\circ} \mathrm{C}$ under a controlled environment, it was affected by these large temperature variations. Improvements to the laser and optical components are under way to make them insensitive to temperature variations.

The performance of the NDL was analyzed by comparing its measurements with an Applanix Inertial Measurement Unit. The Applanix is a high grade instrument using a LN200 inertial navigation system and a sophisticated navigation filter blending the inertial and GPS data. Altitude measurements relative to the ground show excellent agreement with GPS. The flight analyzed and discussed in this report flew above flat as well as hilly terrain several times during repetitive loops of the flight profile. Altitude measurements derived from the three independent LOS range measurements over the different terrains show that error increases linearly with altitude at a rate of approximately $0.2 \%$ of the total altitude when compared with the GPS data processed by the Applanix. The three independent LOS range measurements reduce the impact of terrain features on altitude measurements and thus achieving accuracies that are significantly better than that of a single beam laser altimeter.

When comparing velocity measurements, there is excellent agreement between the NDL and the Applanix during the flight. At times there is a small drift observed between the Applanix and the NDL. This drift is very small, and due to its high degree of correlation to pitch angle, the drift is attributed to the physical separation between the Applanix and NDL to each other and to the center of mass of the helicopter. A discrepancy analysis between the two instruments show that measurement error of the NDL increases with altitude at an approximate rate of .0055\% of the altitude. As an example, at an altitude of 1800 meters, the rms discrepancy due to Doppler frequency measurement amounts to $10 \mathrm{~cm} / \mathrm{sec}$. Comparison of the velocity vector components show excellent agreement between the two instruments, including mean measurement discrepancies of under one-centimeter per second in $\mathrm{x}$ and y- axis (horizontal velocity), and $2.75 \mathrm{~cm} / \mathrm{sec}$ in z-axis (vertical velocity). A total mean velocity magnitude discrepancy of $3.5 \mathrm{~cm} / \mathrm{sec}$ is observed for Flight 4 .

Attitude measurements of the NDL are shown to be of very high quality, approaching the accuracies of the Applanix. Discrepancies in pitch and roll angles are very small during most of the flight, obtaining mean discrepancies of $10.7 \mathrm{mrad}$ in pitch, and $15.7 \mathrm{mrad}$ in roll. The standard deviations are $4.0 \mathrm{mrad}$ in pitch and 6.8 mrad in roll. It has not yet been determined how much of the mean discrepancy values are due to ground topography, and how much is due to sensor placement.

The sensor operated well up to the helicopter flight ceiling, which was approximately 1700 meters. Individual LOS range measurements at this altitude go beyond 2400 meters slant range. It is estimated that when the NDL optical head center axis aligns with nadir, that the current operational range of the sensor is approximately 2000 meters in altitude above ground level. If needed, the operational range of the NDL can be extended by increasing the transmitter output power, improving the receiver SNR by keeping the noise floor low while adding gain, or increasing the diameter of the optical telescopes.

\footnotetext{
i Pierrottet, D., F. Amzajerdian, L. Petway, B. Barnes, G. Lockard,’Flight test performance of a high precision navigation Doppler Lidar”, SPIE Proc. No 7323-34, 2009
} 